\title{
A Prevenção da Osteoporose Levada a Sério: uma Necessidade Nacional
}

\section{carta ao editor}

\section{Marise LazAretti-CAstro SERGIO RAGI EIS João Francisco Marques Neto}

Sociedade Brasileira para o Estudo do Metabolismo Ósseo e Mineral (Sobemom)e Departamento de Metabolismo Ósseo da SBEM (MLC); Sociedade Brasileira de Densitometria Clínica (SBDens)

(SRE); Sociedade Brasileira de Osteoporose (Sobrao) (JFMN), Brasil.

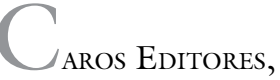

O envelhecimento da população é uma realidade visível ao olho densitométrico e sentida no próprio osso. Esta modificação é de caráter biossocial, óbvia e palpável, confirmada por dados históricos, demonstrando que, nos últimos 26 anos, em nosso País testemunhamos um ganho de dez anos na expectativa de vida ao nascer (1). Este envelhecimento traz consigo, entretanto, aumento alarmante de comorbidades, sendo a osteoporose uma delas. As fraturas são suas temidas conseqüências, que, em si, não causam diretamente a morte, mas que se associam ao grande aumento da morbimortalidade. Uma evidente deterioração da qualidade de vida associada ao incremento da mortalidade, especialmente por causas infecciosas e cardiovasculares, torna a osteoporose um crescente problema de saúde pública.

A perda óssea inexorável que se segue a partir da meia-idade torna passível de desenvolver osteoporose todos aqueles indivíduos que pretendem (e conseguem) superar os 70 anos de idade. Podemos dizer que a instalação da osteoporose é uma questão de tempo; quem viver o suficiente a desenvolverá. Portanto, a prevenção das fraturas osteoporóticas deve fazer parte da estratégia para o nosso envelhecer bem-sucedido.

A osteoporose possui, como substrato fisiopatológico, alterações hormonais e imunológicas que se entrelaçam sobre um pano de fundo genético e ambiental, o que torna o endocrinologista um dos especialistas mais competentes para conduzir sua prevenção, seu diagnóstico e seu tratamento. A endocrinologia encontra-se ainda entre as poucas especialidades médicas reconhecidas pela Associação Médica Brasileira $(\mathrm{AMB})$ para exercer a função de densitometrista, isto é, realizar e laudar exames de densitometria óssea.

A medida da densidade mineral óssea correlaciona-se fortemente com o risco de fratura, mas sabemos que a interpretação de seus resultados depende de uma série de fatores, como idade, sexo, índice de massa corporal (IMC), hábitos de vida, uso de medicamentos, presença de comorbidades, entre outros. No último dia 21 de fevereiro, uma edição especial do periódico Osteoporosis International trouxe uma série de artigos fundamentados no instrumento FRAX® (WHO Fracture Risk Assessment Tool), um modelo que fornece o risco absoluto de fratura nos próximos dez anos para determinado paciente (2). Este programa foi desenvolvido pela comissão de especialistas em nome da Organização Mundial da Saúde (OMS), com base em "meganálises" de estudos epidemiológicos feitos anteriormente na Europa, na Austrália, nos Estados Unidos e na Ásia (3). No mesmo suplemento encontram-se ainda artigos, nos quais adequações do modelo foram feitos para o Japão (4), Reino Unido (5) e Estados Unidos (6). Segundo a análise americana, por exemplo, aponta-se o custo-efetividade do tratamento para osteoporose quando o risco de fratura nos próximos dez anos atingir 3\%, e este grupo de pesquisadores 
ainda conclui que o método FRAX será útil para a tomada de decisão terapêutica (7).

Em poucas palavras, esta ferramenta associa os valores de densidade mineral óssea a outros oito fatores de risco em equações de análise multivariada que calculam o risco absoluto de um indivíduo sofrer, nos próximos dez anos, uma fratura do colo femoral ou outras fraturas osteoporóticas maiores (quadril, vertebrais sintomáticas, úmero proximal e antebraço distal). Como esses eventos estão fortemente associados à utilização de recursos de saúde, direta ou indiretamente, essa nova abordagem procura correlacionar os custos necessários, a fim de evitar fraturas ou, ainda, para ganhar anos de vida qualitativamente úteis.

No futuro próximo, os equipamentos de densitometria central (DXA) proporcionarão relatórios com a informação do risco absoluto de fraturas, incorporando este novo algoritmo da OMS em seus tradicionais laudos. Os médicos já podem digitar na Internet os valores do T-score de densitometrias de fêmur proximal e demais dados relativos aos fatores de risco para obter, em segundos, o risco absoluto estimado de fraturas em dez anos (www.shef.ac.uk/frax). Neste site é possível se obter o risco de fraturas específico para nove países, entre eles Reino Unido, França, Espanha, Itália, Turquia, Suécia, Japão, China e Estados Unidos, e isso só foi possível porque nestes países existiam dados epidemiológicos consistentes.

A determinação do risco absoluto deve ser, preferencialmente, obtida para cada país ou comunidade, com base em estudos de epidemiologia clínica, na medida de suas características genéticas e ambientais. Estudos de farmacoeconomia também são fundamentais, pois organizam o potencial de recursos financeiros disponíveis para a saúde, que variam fortemente de um país para outro.

Por hora, em terras brasileiras, esta deve ser nossa lição de casa: definir nosso risco de fratura e a relação de custo-efetividade dos diferentes tratamentos para a osteoporose sob a ótica de nossa realidade econômica. Além disso, há de se determinar qual o ponto de corte adequado à realidade brasileira para que pacientes de risco real possam ter indicação e acesso aos tratamentos diante dos nossos próprios dados epidemiológicos.
Compreender esse novo modelo e responder a essas perguntas são, desde já, desafios que certamente ocuparão nossas pautas nos próximos anos, até que possamos adequar esse modelo à nossa prática. Para que isso aconteça, conclamamos todas as associações de profissionais relacionados com o tema osteoporose que se apresentem e sugiram propostas para iniciar o debate. Em nome das sociedades que representamos, propomos iniciar os trabalhos já. Para tal, será fundamental o apoio da recém-criada Confederação das Entidades Brasileiras de Osteoporose e Osteometabolismo (Cebom).

Reconhecemos que nosso caminho será longo, pois, apesar de todos os avanços no conhecimento desta doença e na efetividade de seu tratamento, precisamos vencer ainda a realidade adversa, na qual, a maioria dos pacientes que já sofreu fraturas osteoporóticas permanece não tratada. Mas temos de dar o primeiro passo, e esperamos a colaboração de todos os interessados em metabolismo ósseo e epidemiologia clínica para juntos melhorarmos a prevenção contra a osteoporose.

\section{REFERÊNCIAS}

1. Disponível em: http://www.ibge.gov.br/home/estatistica/populacao.

2. Disponível em: http://www.shef.ac.uk/FRAX/index.htm.

3. Siris E; Delmas P. Assessment of 10-year absolute fracture risk: a new paradigm with worldwide application. Osteoporosis Int. DOI 10.1007/s00198-008-0564-8 (Editorial).

4. Fujiwara S, Nakamura T, Orimo H, Hosoi T, Gorai I, Oden A, et al. Development and application of a japanese model of the WHO fracture risk assessment tool (FRAXtrade mark). Osteoporos Int. 2008 Apr;19(4):429-35.

5. Kanis JA, Johnell O, Oden A, Johansson H, McCloskey E. FRAXtrade mark and the assessment of fracture probability in men and women from the UK. Osteoporos Int. 2008 Apr;19(4):385-97. Epub 2008 Feb 22.

6. Dawson-Hughes B, Tosteson AN, Melton LJ 3rd, Baim S, Favus MJ, Khosla S, Lindsay RL. Implications of absolute fracture risk assessment for osteoporosis practice guidelines in the USA. Osteoporos Int. 2008 Apr;19(4):449-58.

7. Tosteson AN, Melton LJ 3rd, Dawson-Hughes B, Baim S, Fa vus MJ, Khosla S, et al. Cost-effective osteoporosis treatment thresholds: the United States perspective. Osteoporos Int. 2008 Apr;19(4):437-47. 